\title{
Efeitos da alteração na posição do selim da bicicleta sobre a corrida subsequente
}

\author{
The effects of bicycle seat tube angle on subsequent running
}

1 Universidade do Estado de Santa Catarina.

Centro de Ciências da Saúde e do Esporte. Laboratório de Pesquisas em Desempenho Humano. Florianópolis, SC. Brasil.

Recebido em 14/02/11 Revisado em 19/04/11 Aprovado em 15/06/11
Resumo - O propósito desse estudo foi analisar os efeitos da alteração no ângulo do tubo do selim (ATS) da bicicleta sobre variáveis fisiológicas e biomecânicas, durante o ciclismo e no desempenho de corrida subsequente. Seis triatletas amadores do sexo masculino $(24,1 \pm 7,8$ anos; $71,3 \pm 7,6 \mathrm{~kg} ; 1,76 \pm 4,8 \mathrm{~m})$ submeteram-se, em dias diferentes e aleatórios, a dois testes de $20 \mathrm{~km}$ de ciclismo (um a $73^{\circ}$ e outro a $80^{\circ}$ ATS) em uma intensidade autosselecionada, em suas próprias bicicletas, acopladas a um ciclossimulador com transição imediata para o desempenho de corrida na distância de $3 \mathrm{~km}$. A intensidade autosselecionada, no primeiro teste de ciclismo, foi fixada durante o segundo teste. Não foram observadas diferenças significantes durante o ciclismo entre os diferentes ATS. Durante a corrida, a velocidade do $2^{\circ} \mathrm{km}$ foi significativamente maior no ângulo de $80^{\circ}(3,99 \pm 0,44 \mathrm{~m} / \mathrm{s})$ comparado ao de $73^{\circ}(3,92 \pm 0,45 \mathrm{~m} / \mathrm{s})$, sem diferença significativa $(P=0,065)$ entre os ângulos na velocidade média total $\left(4,07+0,46 \mathrm{~m} / \mathrm{s}\right.$ a $73^{\circ}$ e $4,18+0,47 \mathrm{~m} / \mathrm{s}$ a $\left.80^{\circ}\right)$. Houve um aumento significativo na frequência de passada a $73^{\circ}$ quando comparado a $80^{\circ}$ ATS no $3 \circ \mathrm{km}(88,2 \pm 0,12$ passadas $/ \mathrm{min}$ e $91,2 \pm 0,12$ passadas/min, respectivamente) e na média total da corrida $(87,6 \pm 0,12$ passadas/ min para 90,0 $\pm 0,08$ passadas/min, respectivamente). Dessa forma, podemos concluir que as alterações no ATS não modificaram significantemente as variáveis fisiológicas e biomecânicas, durante o ciclismo, assim como o rendimento dos $3 \mathrm{~km}$ de corrida subsequentes. Entretanto, as diferenças observadas na mecânica, no ritmo da corrida e a tendência $(P=$ $0,065)$ de melhora do desempenho indicam um pequeno mais significativo efeito sobre a corrida realizada após o ciclismo com ATS mais elevado, aspectos que podem ser relevantes para o rendimento final em uma competição.

Palavras-chave: Ciclismo; Corrida; Engenharia humana; Desempenho atlético; Metabolismo energético.

Abstract - The objective of this study was to investigate the effects of two different $\left(73^{\circ}\right.$ and $80^{\circ}$ ) seat tube angles (STAs) on physiological and biomechanical variables during cycling and on subsequent running performance. Six male triathletes $(24.1 \pm 7.8$ year; $71.3 \pm 7.6 \mathrm{~kg} ; 1.76 \pm 4.8$ m) completed two $20-\mathrm{km}$ cycling trials $\left(\right.$ at $73^{\circ}$ or $80^{\circ}$ ) at self-selected intensity on their own bikes using a cycle simulator and immediately transitioned to a $3-\mathrm{km}$ running trial on a track at maximum pace. The trials were performed randomly on different days. The self-select intensity adopted in the first $20-\mathrm{km}$ cycling trial was also used in the second cycling trial. There were no significant differences between the $73^{\circ}$ and $80^{\circ}$ STAs during the cycling trials. The pace during the $2 \mathrm{nd} \mathrm{km}$ of the running trial was significantly lower at $73^{\circ}(3.92+0.45 \mathrm{~m} / \mathrm{s})$ compared with $80^{\circ}(3.99+0.44 \mathrm{~m} / \mathrm{s})$; however, there was no significant difference $(P=0.065)$ between the STAs in terms of total mean pace $\left(4.07+0.46 \mathrm{~m} / \mathrm{s}\right.$ at $73^{\circ}$ and $4.18+0.47 \mathrm{~m} / \mathrm{s}$ at $\left.80^{\circ}\right)$. The stride rate $(88.2+0.12$ stride $/ \mathrm{min})$ and total mean $(87.6+0.12$ stride/min) during the $3 \mathrm{rd} \mathrm{km}$ of the running trial were significantly lower at $73^{\circ}$ compared with $80^{\circ}(91.2+0.12$ stride/min and $90.0+0.08$ stride/min, respectively). Therefore, the changes in the STAs did not affect significantly the physiological and biomechanical variables during cycling neither subsequent 3-km running performance. However, the differences found in the stride rate, running pace, and tendency of better running performance $(P=0.065)$ suggest a small but significant effect on the running trial performed after cycling using higher STA. These aspects may have a relevant influence on the final race classification.

Key words: Athletic performance; Bicycling; Energy metabolism; Human Engineering; Running. 


\section{INTRODUÇÃO}

O triatlo é uma modalidade de longa duração, no qual o atleta compete de modo sequencial na natação, ciclismo e corrida. Um importante aspecto no triatlo seria o efeito que uma modalidade exerce sobre a outra e consequentemente, no desempenho final de uma competição. Há um empirismo por parte dos praticantes da modalidade, de que mudanças na geometria da bicicleta podem melhorar o desempenho na corrida. Uma alteração comum na geometria da bicicleta é a utilização de um suporte de selim ou até mesmo de bicicletas que permitem pedalar com um ângulo do tubo do selim (ATS) mais acentuado $\left(\sim 80^{\circ}\right)$. O ATS de uma bicicleta é definido na intersecção do tubo do selim com uma linha paralela ao solo. Um maior ATS permite mais conforto e eficiência em posição aerodinâmica com antebraços repousados sobre o guidom aerodinâmico ${ }^{1,2}$. Este aspecto poderia resultar numa transição mais eficiente para a etapa de corrida e consequentemente, melhora do rendimento. No entanto, há poucas evidências científicas que sustentem essa premissa.

Estudos anteriores encontraram diferenças significativas no gasto energético em diferentes ATS, com as maiores angulações $\left(>80^{\circ}\right)$, sendo mais eficientes (i.e. menor $\mathrm{VO}_{2}$ ) comparadas às menores angulações $\left(<70^{\circ}\right)^{1,2}$. Porém, nestes estudos os indivíduos não utilizaram guidões aerodinâmicos (aero-bar), comumente usados em provas de triatlo. Demonstrando a influência da posição aerodinâmica, Gnehm et al. ${ }^{3}$ estimaram uma economia de até 100W em ciclistas profissionais, utilizando guidões aerodinâmicos (posição aerodinâmica) comparado à posição tradicional (postura mais ereta e menor ATS). Por outro lado, ao pedalar em uma posição aerodinâmica, os ciclistas demonstram um maior gasto energético quando a resistência do ar não foi levada em consideração ${ }^{3,4}$. Esse aumento no gasto energético parece ser dependente da especificidade do treinamento ${ }^{4,5}$, uma vez que os triatletas, os quais treinam periodicamente em posições aerodinâmicas, não demonstraram diferenças no gasto energético quando foram comparados nas diferentes posições.

Especificamente em relação ao triatlo, poucos trabalhos têm investigado os possíveis efeitos da alteração no ATS sobre o rendimento na corrida subsequentemente. Garside e Doran ${ }^{6}$ avaliaram o efeito de dois diferentes ATS $\left(73^{\circ}\right.$ e $\left.81^{\circ}\right)$ sobre o desempenho de $10 \mathrm{~km}$ de corrida e encontraram uma melhora significativa no desempenho da cor- rida pedalando na angulação de $81^{\circ}$ ATS. Além disso, os autores observaram que a melhora no desempenho de corrida ocorreu durante a primeira metade dos $10 \mathrm{~km}$ de corrida. Entretanto, neste estudo os sujeitos pedalaram em uma intensidade pré-determinada $\left(70 \% \mathrm{VO}_{2} \max \right)$, o que difere da estratégia de ritmo e intensidade adotada durante uma competição de Triatlo. Além disso, as modificações no ATS e altura do guidão provavelmente modificaram os ângulos do tronco, quadril e joelho. Tentando isolar esses aspectos, Jackson et al. ${ }^{7}$ após modificarem o ATS $\left(73^{\circ}\right.$ e $\left.81^{\circ}\right)$ mantendo constante os ângulos do tronco, quadril e joelho, não encontraram alterações fisiológicas significantes tanto durante o ciclismo (ATS $73^{\circ}$ vs. $81^{\circ}$ ) quanto durante a corrida subsequente. Apesar deste último estudo ter utilizado ritmos autosselecionados durante o ciclismo, a velocidade de corrida foi fixa entre os dois testes, o que não permitiu observar o efeito da alteração do ATS sobre o desempenho de corrida.

Assim, ainda permanecem lacunas sobre os possíveis efeitos das diferentes geometrias da bicicleta no rendimento do Triatlo devido à grande variedade de protocolos adotados. A maior parte dos estudos encontrados na literatura, envolvendo a avaliação da técnica de pedalada, utiliza cargas fixas e iguais para todos os sujeitos ${ }^{6,8}$. Entretanto, estas cargas podem sub ou superestimar a intensidade autosselecionada pelos avaliados durante uma competição. Além disso, quando o objetivo é avaliar modificações no desempenho seria interessante tentar aproximar o máximo possível de uma condição real de competição. Nesse sentido, o objetivo do presente estudo foi analisar os efeitos do ATS da bicicleta sobre variáveis fisiológicas durante o ciclismo e no desempenho de corrida subsequente em triatletas, adotando uma intensidade autosselecionada em ambos, ciclismo e corrida, tornando a condição mais próxima a de uma competição de triatlo. A nossa hipótese é que tornando as condições mais próximas à competição, o menor gasto energético associado ao maior $\operatorname{ATS}^{1,2}\left(80^{\circ}\right)$ poderá promover uma melhora no desempenho da corrida no triatlo.

\section{PROCEDIMENTOS METODOLÓGICOS}

\section{Amostra}

Participaram do estudo 6 triatletas do sexo masculino $(24,16 \pm 7,8$ anos; $176 \pm 4,8 \mathrm{~cm} ; 71,3+7,6 \mathrm{~kg})$, com experiência mínima de 3 anos em competições estaduais e nacionais e que não faziam uso 
regular de qualquer tipo de medicamento. Todos foram informados textual e verbalmente sobre os objetivos e os métodos desse estudo, e assinaram um termo de consentimento livre e esclarecido. Os procedimentos desse estudo foram aprovados pelo Comitê de Ética em Pesquisas com Seres Humanos da Universidade na qual o estudo foi realizado (Protocolo 166/2009).

Os sujeitos foram orientados a se apresentarem para os testes descansados, alimentados, hidratados e a não realizarem esforços intensos nas 48 horas antecedentes aos mesmos. Os testes foram realizados no mesmo local e horário do dia.

\section{Protocolo de teste}

Foram realizadas duas sessões experimentais, em ordem aleatória, com intervalos de 72 hs. Os testes consistiam em $20 \mathrm{~km}$ de ciclismo, a $73^{\circ}$ ou $80^{\circ}$ ATS, seguidos por $3 \mathrm{~km}$ de corrida máxima. Os ATS foram ajustados para $73^{\circ}$ ou $80^{\circ}$, utilizando diferentes canotes, um reto que posicionava o selim a $73^{\circ}$ e outro com uma inclinação que proporcionava o ângulo de $80^{\circ}$. Em função das diferentes geometrias entre os quadros, ajustes foram realizados, movimentando o selim para frente ou para trás sobre seu trilho para que fosse atingido o ângulo predeterminado $\left(73^{\circ}\right.$ ou $\left.80^{\circ}\right)$. O Ângulo foi mensurado através de um goniômetro, considerando como referência o ponto no qual a reta entre o trocanter do ciclista e o eixo do movimento central da bicicleta perfazia o ângulo predeterminado com uma reta paralela ao solo.

Os atletas realizaram os $20 \mathrm{~km}$ na própria bicicleta acoplada a um ciclossimulador (Computrainer Pro, Racer Mate, Seattle, USA) utilizando um software específico (Computrainer Coaching Software, Racer Mate, Seattle, USA). Os 3 km correndo foram realizados em uma pista de 200 metros de atletismo sintética ao ar livre. Todos os triatletas realizaram ambos os testes individualmente, sendo também instruídos a completar o teste $(20 \mathrm{~km}$ pedalando e $3 \mathrm{~km}$ correndo) o mais rápido possível. Durante os primeiros $3 \mathrm{~km}$ do ciclismo, os triatletas autosselecionaram uma velocidade e cadência que foi mantida durante os $17 \mathrm{~km}$ restantes. No outro dia de teste, o ciclismo foi realizado com a mesma velocidade e cadência de pedaladas determinadas no teste anterior. A frequência cardíaca foi mensurada continuamente durante todos os testes (Polar S610i, Polar, Kempele, Finlândia). Em ambos os testes, foram coletadas amostras de sangue do lóbulo da orelha no repouso, no $5^{\circ}, 10^{\circ}, 15^{\circ}$ e $19^{\circ}$ $\mathrm{km}$ do ciclismo, e ao final da corrida.
Através do Computrainer Coaching Software, foram registradas as seguintes informações: Velocidade $(\mathrm{km} / \mathrm{h})$; Potência (W) e Cadência (rpm), a cada $1,5 \mathrm{~s}$ e, posteriormente reduzidas a valores médios do último quilometro para o $5^{\circ}, 10^{\circ}, 15^{\circ}$ e $19^{\circ} \mathrm{km}$.

\section{Determinação do lactato sanguíneo}

Para a determinação do lactato sanguíneo, foram coletados do lóbulo da orelha, sem hiperemia, $25 \mu \mathrm{l}$ de sangue em capilar heparinizado, sendo, a seguir, imediatamente transferido para microtubos de polietileno com tampa - tipo Eppendorff - de 1,5 ml, contendo $50 \mu$ de solução de $\mathrm{NaF} 1 \%$ e armazenado em gelo. A análise do lactato foi realizada através de analisador eletroquímico modelo YSI 1500 (Yelow Spring Instruments, USA).

\section{Análise da cinemática da corrida}

A frequência de passada (FP), comprimento de passadas (CP) foram determinados em todos os testes de corrida a partir de vídeos registrados em uma filmadora (Sanyo VPC-CA65), em um trecho reto de $20 \mathrm{~m}$ previamente marcado com cones. A FP foi calculada utilizando o tempo necessário para se realizar cinco ciclos completos de passadas, e expressa em frequência de passadas por minuto. $\mathrm{O}$ $\mathrm{CP}$ foi calculado dividindo a velocidade média do trecho de $20 \mathrm{~m}$ pela FP.

\section{Análise Estatística}

Foram calculados as médias e desvios padrões dos dados obtidos. A normalidade dos dados foi verificada através do teste de Shapiro-Wilk. As comparações entre os ângulos foram realizadas pelo Test- $t$ Student para dados pareados. Em todos os testes foi adotado um nível de significância de $p \leq 0,05$.

\section{RESULTADOS}

A tabela 1 apresenta valores médios e desvio padrão das variáveis biomecânicas e fisiológicas durante o ciclismo com ATS de $73^{\circ}$ e $80^{\circ}$. Não foram observadas diferenças significantes entre os diferentes ângulos do selim para todas as variáveis analisadas no presente estudo. Na figura 1, é demonstrado o comportamento do lactato sanguíneo durante o ciclismo com ATS de $73^{\circ}$ e $80^{\circ}$. Não houve diferenças na concentração de lactato ao longo do teste, para cada uma das condições estudadas, como também entre as condições. 
Tabela 1. Valores médios e desvio padrão das variáveis fisiológicas e biomecânicas nos diferentes ângulos do selim obtidos durante os $20 \mathrm{~km}$ de ciclismo.

\begin{tabular}{lcc}
\hline \multirow{2}{*}{ Variável } & \multicolumn{2}{c}{ Ângulo do tubo do selim } \\
\cline { 2 - 3 } & $73^{\circ}$ & $80^{\circ}$ \\
\hline Potência (W) & $219 \pm 24,0$ & $212 \pm 22,6$ \\
Cadência (rpm) & $94,8 \pm 4,7$ & $94,7 \pm 4,6$ \\
FC (bpm) & $162 \pm 10$ & $160 \pm 9$ \\
Lactato sanguíneo (mMol/L) & $5,7+2,3$ & $4,9 \pm 2,8$ \\
\hline
\end{tabular}

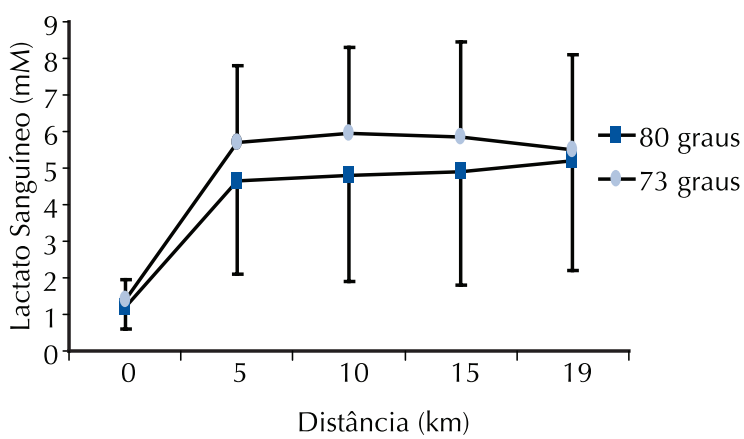

Figura 1. Valores médios e desvio padrão do comportamento do lactato sanguíneo durante o ciclismo com ATS de $73^{\circ}$ e $80^{\circ}$.

Na tabela 2, foram apresentadas as variáveis biomecânicas determinados durante a corrida realizada após o ciclismo com ATS de $73^{\circ}$ e $80^{\circ}$. Observou-se que a velocidade média foi maior no $2^{\circ} \mathrm{km}$ do teste com ângulo de $80^{\circ}$, comparado a $73^{\circ}$. Não foram observadas diferenças nas outras velocidades $\left(1^{\circ} \mathrm{e} 3^{\circ} \mathrm{km}\right.$, e velocidade média total) entre os ângulos de $73^{\circ}$ e $80^{\circ}$. A frequência de passadas aumentou significativamente no $3^{\circ} \mathrm{km}$ e no total da corrida do teste com ângulo de $80^{\circ}$ comparado a $73^{\circ}$. Não foram observadas diferenças no $\mathrm{CP}$ em todas as condições analisadas. Os valores de pico da FC $(185,8 \pm 5,3$ e $184,3 \pm 7,9$ bpm $)$ e [La] $(10,7 \pm 1,5$ e $10,1 \pm 2,4 \mathrm{mMol} / \mathrm{L})$ durante a corrida não foram diferentes entre $73^{\circ}$ e $80^{\circ}$, respectivamente.

Tabela 2. Médias \pm DP das variáveis biomecânicas obtidas durante o teste de $3 \mathrm{~km}$ de corrida realizada logo após o ciclismo. $1 \mathrm{~km}$, média obtida no $1^{\circ} \mathrm{km} ; 2 \mathrm{~km}$, média obtida no $2^{\circ} \mathrm{km}$; $3 \mathrm{~km}$, média obtida no $3^{\circ} \mathrm{km}$; Total; média obtida nos $3 \mathrm{Km}$ totais; FP, frequência de passada; CP, comprimento de passada.

\begin{tabular}{ccccc}
\hline & & $\begin{array}{c}\text { Velocidade } \\
(\mathrm{m} / \mathrm{s})\end{array}$ & $\begin{array}{c}\mathrm{FP} \\
(\text { Passada/min })\end{array}$ & $\begin{array}{c}\mathrm{CP} \\
(\mathrm{m})\end{array}$ \\
\hline \multirow{2}{*}{$1 \mathrm{~km}$} & $73^{\circ}$ & $4,15 \pm 0,52$ & $87,6 \pm 0,14$ & $2,84 \pm 0,30$ \\
& $80^{\circ}$ & $4,22 \pm 0,5$ & $91,2 \pm 0,10$ & $2,78 \pm 0,31$ \\
\multirow{3}{*}{$2 \mathrm{~km}$} & $73^{\circ}$ & $3,92 \pm 0,45$ & $86,4 \pm 0,08$ & $2,72 \pm 0,28$ \\
& $80^{\circ}$ & $3,99 \pm 0,44^{*}$ & $88,2 \pm 0,04$ & $2,72 \pm 0,28$ \\
\multirow{3}{*}{$3 \mathrm{~km}$} & $73^{\circ}$ & $4,14 \pm 0,43$ & $88,2 \pm 0,12$ & $2,82 \pm 0,23$ \\
& $80^{\circ}$ & $4,32 \pm 0,47$ & $91,2 \pm 0,12^{*}$ & $2,85 \pm 0,27$ \\
\multirow{3}{*}{ Total } & $73^{\circ}$ & $4.07 \pm 0,46$ & $87.6 \pm 0,12$ & $2,79 \pm 0,26$ \\
& $80^{\circ}$ & $4,18 \pm 0,47^{*}$ & $90,0 \pm 0,08^{*}$ & $2,78 \pm 0,27$ \\
\hline
\end{tabular}

* p<0,05 em relação ao $73^{\circ}$ na mesma situação. \# p=0,065 em relação ao $73^{\circ}$

\section{DISCUSSÃO}

O presente estudo apresenta relevância na escolha do equipamento mais adequado para triatletas. A proposta inicial das avaliações, de um ritmo auto-selecionado no ciclismo e corrida máxima proporcionou maior similaridade ao teste comparado com a dinâmica de uma prova de triatlo. Tal aspecto diferencia-se dos estudos anteriores que usaram cargas pré determinadas. Mesmo utilizando situações mais próximas à competição, nossa hipótese inicial foi parcialmente confirmada, uma vez que o aumento do ATS causou modificações na técnica (FP) e no ritmo (maior velocidade no segundo quilometro) da corrida, com uma tendência de melhora no desempenho total da corrida.

No presente estudo, não foram observadas diferenças fisiológicas significantes durante o ciclismo entre os ATS de $73^{\circ}$ e $80^{\circ}$. Estes achados corroboram os resultados encontrados por outros estudos ${ }^{6,7}$. Com relação às respostas fisiológicas, a estabilidade observada nas concentrações de lactato sanguíneo ao longo dos $20 \mathrm{~km}$ de ciclismo (aumentos menores que $1 \mathrm{mM}$ entre o $5^{\circ}$ e $19^{\circ} \mathrm{km}$, Figura 1), indicam que os atletas pedalaram dentro do domínio pesado de exercício. O domínio pesado compreende exercícios realizados a partir da menor intensidade de esforço no qual o lactato se eleva em relação aos valores da linha de base (i.e. Limiar de Lactato) e tem como limite superior a intensidade correspondente à máxima fase estável de lactato ou a Potência crítica9. Estas respostas corroboram os dados de Denadai e Balikian ${ }^{10}$ os quais demonstraram que durante os $20 \mathrm{~km}$ de ciclismo em uma competição de triatlo os indivíduos pedalaram na intensidade abaixo do Limiar Anaeróbio. Portanto, o nosso objetivo de simular uma intensidade próxima de uma competição parece ter sido alcançada. Um fator limitante deste estudo foi a ausência de medidas diretas do $\mathrm{VO}_{2}$, que poderia de modo mais preciso indicar possíveis modificações no gasto energético durante o ciclismo entre os diferentes ATS. No entanto, estudos anteriores que objetivaram observar o efeito de modificações no ATS sobre a corrida subsequente não encontraram alterações no $\mathrm{VO}_{2}$ durante o ciclismo nas diferentes condições ${ }^{6,7}$.

Tem sido demonstrada uma melhora no desempenho da corrida no triatlo quando a carga do ciclismo foi reduzida através da diminuição da resistência do ar (pedalando atrás de outro ciclista) ${ }^{11}$ com o uso dos guidões aerodinâmicos ${ }^{12}$, modificações na cadência de pedalada ${ }^{13}$, intensidade mantida constante ao longo do teste ${ }^{14}$ ou com o ATS 
acentuado $\left(81^{\circ}\right)^{6}$. Neste último estudo, foi verificada uma possível estratégia de corrida progressiva após pedalar com o ATS de $73^{\circ}$, sugerindo que embora essa estratégia possa levar a um pior desempenho inicial, este poderia ser compensado permitindo que o atleta corra os próximos quilômetros em um ritmo mais rápido, possibilitando uma melhora do desempenho final de prova ${ }^{15}$. Entretanto, os sujeitos iniciaram os primeiros $5 \mathrm{~km}$ de corrida com uma maior velocidade após pedalar com o ATS de $80^{\circ}$, comparado ao ATS de $73^{\circ}$, levando a uma melhora no desempenho dos $10 \mathrm{~km}$ com o ATS mais eleva$\mathrm{do}^{6}$. Assim, a vantagem de um ângulo mais elevado seria permitir aos triatletas iniciar a etapa de corrida mais rapidamente, alcançando, assim, uma média de velocidade superior a comparada a uma menor angulação ${ }^{6}$. Na tentativa de corrigir uma possível limitação do estudo de Garside e Doram ${ }^{6}$, que foi a utilização da mesma intensidade relativa para todos os sujeitos durante o ciclismo, Jackson et al. ${ }^{7}$ elaboraram um teste combinado de ciclismo e corrida com ritmo autosselecionado em ambas as modalidades (próximo ao de competição), e não encontraram mudança fisiológica significativa durante a corrida. Apesar da adoção de um ritmo autosselecionado, o desenho experimental utilizado não permitiu aos autores observar efeitos da alteração do ATS sobre o rendimento de corrida, uma vez que a velocidade autosselecionada foi fixada nos dois testes de corrida?

Em nosso estudo, foi adotado uma intensidade autosselecionada no ciclismo e máximo na corrida, a fim de investigar alterações significativas no desempenho da corrida em triatletas. A maior velocidade observada no segundo quilômetro da corrida, após pedalar com o ATS de $80^{\circ}$, comparado ao de $73^{\circ}$, sugere uma menor diminuição do ritmo para esse estágio da avaliação, o qual é caracterizado por uma queda na velocidade, comparada aos estágios iniciais $\left(1^{\circ} \mathrm{km}\right)$ de corrida em ambas as condições. Além disso, corroborando, em parte, os achados de Garside e Doran ${ }^{6}$, nós observamos uma tendência $(p=0,065)$ de melhora no desempenho de corrida após pedalar com um ATS de $80^{\circ}$. O uso da geometria com $80^{\circ}$ ATS poderia reduzir os efeitos deletérios do ciclismo sobre a corrida, minimizando alterações no recrutamento muscular, quando comparados com os padrões durante o ciclismo com um ATS de $73^{\circ}$. Reforçando esta possível explicação, Ricard et al. ${ }^{16}$ demonstraram uma diminuição significativa na atividade do músculo bíceps femoral quando o ciclismo foi realizado em um ATS de $82^{\circ}$, comparado ao de $72^{\circ}$. Além disso, Heiden e Burnett ${ }^{17}$ sugeriram que uma redução na ativação do bíceps femoral poderia melhorar a corrida durante o Triatlo. Um aspecto que poderia ajudar a explicar a ausência de diferença estatística significante no tempo total de corrida, para o presente estudo, comparado ao estudo de Garside e Doram ${ }^{6}$, seria a distância total do teste de corrida, $3 \mathrm{~km}$ e 10 $\mathrm{km}$, respectivamente, a qual acarreta diferenças, principalmente, no ritmo adotado, participação dos sistemas energéticos e padrão de recrutamento muscular ${ }^{18}$. Além disso, o baixo número de sujeitos também contribuiu para um menor poder estatístico, o que poderia ter levado a um erro do tipo II.

Mudanças significativas na frequência de passadas foram observadas no teste de corrida com ATS de $80^{\circ}$, quando comparado ao de $73^{\circ}$, sem diferenças no comprimento da passada. Esse tipo de alteração nos padrões técnicos (aumento da frequência de passadas) são comumente observados como estratégia para aumento da velocidade, conforme demonstrado por Garside e Doram ${ }^{6}$ nos primeiros 5 $\mathrm{km}$. Por outro lado, alguns estudos que avaliaram o efeito da fadiga sobre a técnica de corrida observaram uma diminuição no comprimento de passadas, e um aumento na frequência de passadas como uma tentativa de manter a mesma velocidade de deslocamento $^{19,20}$. Como ambas as condições de corrida analisadas no presente estudo foram precedidas pelo ciclismo e não foram observadas alterações no comprimento de passada, o aumento na frequência de passadas observado após pedalar com o ATS de $80^{\circ}$ parece representar uma tentativa de aumento na velocidade (apesar da ausência de significância estatística), sugerindo que a utilização de uma bicicleta com um ATS mais inclinado poderia reduzir os efeitos deletérios do ciclismo sobre a corrida.

Ao avaliar os resultados do nosso estudo, é importante abordar algumas das suas limitações. Provavelmente, o fator mais importante a se considerar é que, sob condições de competição, os atletas experimentam uma grande variedade de topografias e condições ambientais. Estes podem afetar as respostas às mudanças no ATS e infelizmente, são condições difíceis de serem reproduzidas em laboratório. Outra limitação foi a utilização de uma amostra relativamente pequena de triatletas amadores, o que pode ter afetado o poder estatístico e a transferência dos nossos resultados para atletas de diferentes níveis. Além disso, os dados fisiológicos obtidos em situações controladas de laboratório não podem representar com precisão o que ocorreria durante uma competição de triatlo, e especificamente, no modelo experimental utilizado neste estudo não foi realizada a etapa de natação que poderia, também, 
interferir nos resultados ${ }^{18}$. As possíveis alterações na aerodinâmica gerada pela alteração do ATS causariam modificações no custo metabólico, possíveis de serem observadas durante o ciclismo de campo, foram anuladas durante o ciclismo estacionário em laboratório. Portanto, esse possível efeito não pode ser observado no presente estudo.

\section{CONCLUSÃO}

A partir dos resultados obtidos, podemos concluir que a alteração do ATS não afetou as variáveis fisiológicas durante o ciclismo, mas apresentou uma tendência de melhora no rendimento da corrida subsequente ao ciclismo realizado com um maior ATS, pelo menos no grupo de sujeitos estudados. Além disso, as modificações na técnica e no ritmo da corrida indicam um pequeno, mas significativo efeito do ATS de $80^{\circ}$, sugerindo que a utilização de uma bicicleta com um ATS mais inclinado poderia reduzir os efeitos deletérios do ciclismo sobre a corrida, aspectos que podem ser relevantes para o rendimento final em uma competição. Por conseguinte, ao analisar a escolha do ATS, triatletas deverão enfatizar a melhor combinação de aerodinâmica, geração de força, conforto e manuseio, mantendo ângulos similares ao que eles estão habituados nos treinamentos. Outros fatores, tais como topografia do percurso, condições climáticas, antropometria do triatleta, tipo de triatlo (com permissão ou não para pedalar logo atrás de outro atleta), e da duração do evento também podem desempenhar um papel na escolha do ATS.

\section{REFERÊNCIAS BIBLIOGRÁFICAS}

1. Heil DP, Wilcox AR, Quinn CM. Cardiorespiratory responses to seat-tube angle variation during steady-state cycling. Med Sci Sports Exerc 1995; 27(5):730-5.

2. Price D, Donne B. Effect of variation in seat tube angle at different seat heights on submaximal cycling performance in man. J Sports Sci 1997;15(4): 395-402.

3. Gnehm P, Reichenbach S, Altpeter E, Widmer H, Hoppeler H. Influence of different racing positions on metabolic cost in elite cyclists. Med Sci Sports Exerc 1997;29(6) 818-23.

4. Peveler WBP, Smith J, Richardson M. Effects of training in an aero position on metabolic economy. JEPonline 2005;8(1):44-50.

5. Peveler WBP, Smith J, Richardson M. Effects of training in an aero position on anaerobic power output. . JEPonline 2004;7(5):52-6.

6. Garside IDD. Effects of bicycle frame ergonomics on triathlon $10-\mathrm{km}$ running performance. J Sports Sci 2000;18(10):825-33.
7. Jackson K, Malcure J, Duncan R. Effects of Seat-Tube Angle on the Metabolic Cost of the Cycle-Run Transition in Triathletes. JEPonline 2008; 11(1):45-52.

8. Diefenthaeler F, Bini RR, Nabinger E, Laitano O, Carpes FP, Mota CB, et al. Proposta metodológica para a avaliação da técnica da pedalada de ciclistas: estudo de caso. Rev Bras Med Esporte 2008;14(2):16-8.

9. Gaesser GA, Poole DC. The slow component of oxygen uptake kinetics in humans. Exerc Sport Sci Rev 1996;24:35-71.

10. Denadai BS, Balikian Junior, P. Relação entre limiar anaeróbio e "performance" no short triathlon. Rev Paul Educ Fís 1995;9(1):10-5.

11. Hausswirth C, Vallier JM, Lehenaff D, Brisswalter J, Smith D, Millet G. et al. Effect of two drafting modalities in cycling on running performance. Med Sci Sports Exerc 2001;33(3):485-92.

12. Heil DP, Derrick TR, Whittlesey S. The relationship between preferred and optimal positioning during submaximal cycle ergometry. Eur J Appl Physiol Occup Physiol 1997;75(2):160-5.

13. Bernard T, Vercruyssen F, Grego F, Hausswirth C, Lepers R, Vallier JM, et al. Effect of cycling cadence on subsequent $3 \mathrm{~km}$ running performance in well trained triathletes. Br J Sports Med 2003;37(2);154-8: discussion 159.

14. Bernard T, Vercruyssen F, Mazure C, Gorce P, Hausswirth C, Brisswalter J. Constant versus variable-intensity during cycling: effects on subsequent running performance. Eur J Appl Physiol 2007;99(2):103-11.

15. Kreider RBCD, Hammet JB, Cortes CW, William $\mathrm{KW}$. Effects of cycling on running performance in triathletes. Ann Sports Med 1988;3:220-5.

16. Ricard M, Hills-Meyer P, Miller MG, Michael TJ. The effects of bicycle frame geometry on muscle activation and power during a Wingate anaerobic test. J Sports Sci 2006;5:25-32.

17. Heiden T, Burnett A. The effect of cycling on muscle activation in the running leg of an Olympic distance triathlon. Sports Biomech 2003;2(1):35-49.

18. Brandon LJ. Physiological factors associated with middle distance running performance. Sports Med 1995;19(4):268-77.

19. Elliott BC, Roberts AD. A biomechanical evaluation of the role of fatigue in middle-distance running. Can J Appl Sport Sci 1980;5(4):203-7.

20. Hausswirth C, Bigard AX, Guezennec CY. Relationships between running mechanics and energy cost of running at the end of a triathlon and a marathon. Int J Sports Med 1997;18(5):330-9.
Endereço para correspondência
Fabrizio Caputo
Laboratório de Pesquisas em Desempenho Humano Centro de Ciências da Saúde e do Esporte, UDESC.
Rua Pascoal Simone, 358, Coqueiros
88080-350 - Florianópolis, SC.
E-mail: fabrizio.caputo@udesc.br 